\title{
A CONFORMAL INEQUALITY RELATED TO THE CONDITIONAL GAUGE THEOREM
}

\author{
TERRY R. MCCONNELL
}

\begin{abstract}
We prove the inequality $h(x)^{-1} G(x, y) h(y) \leq c G(x, y)+c$, where $G$ is the Green function of a plane domain $D, h$ is positive and harmonic on $D$, and $c$ is a constant whose value depends on the topological nature of the domain. In particular, for the class of proper simply connected domains $c$ may be taken to be an absolute constant. As an application, we prove the Conditional Gauge Theorem for plane domains of finite area for which the constant $c$ in the above inequality is finite.
\end{abstract}

\section{INTRODUCTION AND STATEMENT OF RESULTS}

The study of conditioned Brownian motion in subdomains $D$ of the plane leads to consideration of the ratios $G(x, y) h(y) / h(x)$, where $G$ is the Green function of $D$ and $h$ is a positive harmonic function on $D$. For example, M. Cranston and the author [10] showed that if $D$ has finite area and $\tau$ is the exit time from $D$ of Brownian motion conditioned by the harmonic function $h$, then $\tau$ has finite expectation. This turns out to be equivalent to the inequality

$$
\int_{D} G(x, y) h(y) d y \leq c|D| h(x),
$$

where $c$ is independent of $D, h$, and $x$, and $|D|$ denotes the area of $D$. A number of authors have considered variants, extensions, and applications of this inequality $[3,4,6,9,12,13$, and 15]. The main purpose of this paper is to show that, for a large class of domains, (1.1) may be strengthened to the pointwise inequality,

$$
h(x)^{-1} G(x, y) h(y) \leq c G(x, y)+c, \quad x, y \in D .
$$

The class of domains includes all proper simply connected domains as well as a number of very pathological infinitely connected domains.

Inequality (1.2) is interesting because it shows how the boundary behaviors of $G$ and $h$ are related, and, like (1.1), it places constraints on the behavior of

Received by the editors August 8, 1988. The results of this paper were presented at the 51 st annual meeting of the Institute of Mathematical Statistics, August 18, 1988.

1980 Mathematics Subject Classification (1985 Revision). Primary 60J45, 60J65; Secondary $81 \mathrm{C} 20$.

Key words and phrases. Green functions, conditioned Brownian motion, conditional gauge theorems, Schrödinger operators, positive harmonic functions.

Research supported in part by NSF Grant DMS 87-00802. 
conditioned Brownian motion. To illustrate the latter point we will show how (1.2) implies the conditional gauge theorem for such domains.

Throughout the paper we assume $D$ is a connected open subset of the plane and has a Green function in the classical sense, i.e., the Green function $G$ is required to approach 0 at every boundary point. This is true, for example, if each connected component of the complement of $D$ relative to the Riemann sphere $\mathbf{C}^{*}$ contains at least 2 (hence, a continuum of) points. (Myrberg [17]. Also see [16, p. 179].)

Theorem 1.1. Let $D \neq \mathbf{C}$ be a domain such that for some constant $\eta>0$ we have $\operatorname{diam}(K) \geq \eta$ for each connected component $K$ of $D^{c}$. Then there is a constant $c$ such that for all distinct $x, y$, and $z$ in $D$,

$$
G(x, z)^{-1} G(x, y) G(y, z) \leq c[G(x, y)+G(y, z)]+c .
$$

For $D \neq \mathbf{C}$ simply connected the constant $c$ in (1.3) may be taken to be an absolute constant. In the general case, $c$ depends on the domain.

A related, but less precise, inequality was proved in [11] for bounded Lipschitz domains in $d$ dimensions.

Because inequality (1.3) is conformally invariant, one may apply the theorem to any domain $D$ for which the hypotheses are satisfied by some conformal image of $D$, provided one is careful to preserve the hypothesis $\operatorname{diam}(K) \geq$ (constant).

For the applications below it is useful to note that if $|D|$ is finite then $c$ can be chosen to depend only on $\eta$ and $|D|$ (see Remark 3.2).

Taking limits in (1.3) along sequences of $z$ tending to $\partial D$ we obtain (1.2) for harmonic functions $h$ corresponding to minimal Martin boundary points. After cross multiplication by $h(x)$, the Martin representation shows that (1.2) holds for all $h>0$ harmonic on $D$ :

Corollary 1.2. Let $D$ be as in Theorem 1.1. Then for each positive harmonic function $h$ on $D$ we have

$$
h(x)^{-1} G(x, y) h(y) \leq c G(x, y)+c, \quad x, y \in D .
$$

Here $c$ is the same constant as in (1.3).

This result may be interpreted as a new kind of Harnack inequality in which the condition " $x$ and $y$ belong to a fixed compact subset of $D$ " is replaced by a condition on $G(x, y)$ :

Corollary 1.3. Let $D$ be as in Theorem 1.1 and $\lambda>0$. Then there is a constant $A$ having the same dependence on $D$ as the constant $c$ in (1.3) and which in addition depends on $\lambda$, such that for each positive harmonic function $h$ on $D$,

$$
G(x, y)=\lambda \Rightarrow A^{-1} h(x) \leq h(y) \leq A h(x) .
$$

The condition on the size of the boundary components of $D$ is essential in each of the results above. In $\S 5$ we show that for bounded triply connected 
domains the best constants $c$ in (1.3) tend to infinity as $\eta$ tends to zero. By connecting worse and worse such examples with very thin "tubes" one may easily construct an infinitely connected domain for which (1.3) fails.

Likewise, none of the above results extends to higher dimensions. Indeed, even the weaker result (1.1) fails for bounded simply connected domains in $\mathbf{R}^{d}$, $d \geq 3[10]$.

Following [11], we shall apply Theorem 1.1 to prove a conditional gauge theorem. Let $B(t)$ be Brownian motion and denote by $\tau_{E}$ the first exit time of $B(t)$ from the open set $E$. (If $E=D$ we will omit the subscript.) Let $V$ be a Borel function on $D$ and define the Feynmann-Kac functional $e_{V}(t)=$ $\exp \left\{\int_{0}^{t} V(B(s)) d s\right\}$. The quantity $F(x)=E_{x} e_{V}(\tau)$ was introduced by Chung and Rao [8] who showed that for $V$ bounded and $|D|<\infty$ one has the implication

$$
F\left(x_{0}\right)<\infty \text { for some } x_{0} \in D \Rightarrow \sup _{x \in D} F(x)<\infty .
$$

The $E_{x}$ above denotes expectation relative to Brownian motion started from $x$. We shall write $E_{x}^{h}$ for expectation relative to Doob's $h$-path Brownian motion started from $x$. When $h$ corresponds to a minimal Martin boundary point $z$ we will write this as $E_{x}^{z}$. In this case, the $h$-path process, also denoted $B(t)$, may be viewed as Brownian motion started from $x$ and conditioned to converge to the point $z$ as $t$ tends to $\tau$.

Let $F(x, z)=E_{x}^{z} e_{V}(\tau)$ whenever this is well defined. In [14] N. Falkner proved that for $V$ bounded and $D$ having $C^{2}$ boundary one has an implication analogous to (1.4):

$$
\begin{aligned}
F\left(x_{0}, z_{0}\right) & <\infty \text { for some }\left(x_{0}, z_{0}\right) \in D \times \partial D \\
& \Rightarrow F(\cdot, \cdot) \text { is bounded over } D \times \partial D .
\end{aligned}
$$

The function $F$ has been termed the conditional gauge and results of the form (1.5) conditional gauge theorems (CGT's). This result has been extended in a number of ways, and it has been applied to the study of the potential theory of the Schrödinger operator $-\frac{1}{2} \Delta+V$. (See [1 and 11].) Considerable effort has been expended towards obtaining the CGT for the widest possible class of domains with $V$ belonging to the Kato class $K_{d}$, because of the fundamental role the latter plays in the theory of Schrödinger operators. We shall not define $K_{d}$ here because we are interested in a wider (in the case of bounded domains) class given by (1.6) and (1.7) below. Instead we refer the reader to [1].

For $V \in K_{d}$ the best result to date is that (1.5) holds in the case of bounded Lipschitz domains [11]. In case $d=2$ much more is true.

Theorem 1.4. Let $D$ be a domain having $|D|<\infty$ and otherwise satisfying the same hypotheses as in Theorem 1.1. Suppose $V$ is a Borel function on $D$ satisfying the following pair of conditions:

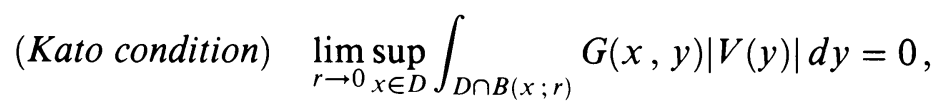


and

$$
V \in L^{1}(D)
$$

Then the implication (1.5) is true.

We remark that each $V$ in $L^{p}(D)$ for $p>1$ satisfies (1.6) and (1.7), and that for bounded domains the class of functions satisfying these conditions includes the usual Kato class $K_{2}$. The CGT fails in general for domains of infinite area. To construct a counterexample, take for $D$ an infinite strip, for $z$ one of the two Martin boundary points at infinity, and for $V$ a sufficiently small constant.

It is also noteworthy that, while [11 and 15] convey the impression that the CGT in higher dimensions is related to smoothness properties of $\partial D$, our hypotheses are of a completely different character. One may even have $|\partial D|>$ 0 -take for $D$ the unit square, less all vertical segments of the form $\{x\} \times$ $[1 / 4,3 / 4]$, where $x$ ranges over a Cantor subset of $[0,1]$ of positive measure.

Finally, we should point out that the result of [11] is more general than (1.5) in that $x_{0}$ is allowed to lie in $\partial D$, with the conclusion being that $F$ is bounded over $\bar{D} \times \bar{D}$. Our result does not extend in this way. Salisbury [18] has constructed a domain $D$ satisfying our hypotheses, and points $x, z \in \partial D$, for which there does not exist a conditioned Brownian motion from $x$ to $z$.

In the next section we prove Theorem 1.1 in the special case of a simply connected domain. Strictly speaking, this is unnecessary since the proof of the general case in $\S 3$ does not depend on having first established the result for simply connected domains. However, we feel that the proof is instructive, and moreover it leads to a reasonable value for the constant $c$ (6.1 will work). Theorem 1.4 is proved in $\S 4$, and $\S 5$ contains the example alluded to in the discussion following Corollary 1.3.

\section{THE SIMPLY CONNECTED CASE}

Let $D$ be a simply connected subset of $\mathbf{C}^{*}$. By performing an inversion if necessary we may assume that $D$ is a proper simply connected subdomain of C. We shall prove

$$
G(x, z)^{-1} G(x, y) G(y, z) \leq c_{1}[G(x, y)+G(y, z)]+c_{2}
$$

with $c_{1}=\frac{5}{2} \log 3$ and $c_{2}=8 \log 2 \log 3$.

By the Riemann mapping theorem we may assume $D$ is the unit disc, $x=0$, and $0<y<1$. In this case $G(y, z)=-\log |z-y|+\log \left|1-z^{*} y\right|$, where $z^{*}$ denotes the complex conjugate of $z$, and (2.1) reduces to

$$
(\log |z|)^{-1} G(y, z) \log y \leq c_{1}[-\log y+G(y, z)]+c_{2} .
$$

Clearly, we may always assume $y<|z|$.

We shall consider the following four cases separately:

Case 1. $y<|z|<1 / 2$,

Case 2. $|z| \geq 1 / 2,1 / 4<y,|z|^{2}<y$, 
Case 3. $|z| \geq 1 / 2,1 / 4<y \leq|z|^{2}$, and

Case 4. $|z| \geq 1 / 2, y \leq 1 / 4$.

In Case 1, first suppose $|y-z| \leq|z| / 2$. Then $y \geq|z| / 2$ so that $(\log |z|)^{-1} G(y, z) \log y \leq 2 G(z, y)$. On the other hand, if $|y-z|>|z| / 2$ then $-\log |y-z| \leq \log 2-\log |z|$. Since $G(y, z)<\log (5 / 4)-\log |y-z|$ we conclude that $(\log |z|)^{-1} G(y, z) \log y<(\log 5) / \log 2 \log (1 / y)$.

In case 2 we have

$$
(\log |z|)^{-1} G(y, z) \log y \leq(\log |z|)^{-1} G(y, z) \log \left(|z|^{2}\right)=2 G(y, z) .
$$

As for Case 3, we will prove that

$$
(\log |z|)^{-1} G(y, z) \log y \leq 8 \log 2 \log 3 .
$$

It is well known that $G(z, y) \leq G(|z|, y)$. (This may be checked directly. It also follows from a general result about circularly symmetric domains [2,

p. 154].) Therefore, in proving (2.3) we may assume that $1 / 4<y \leq z^{2}<z<$ 1 . From the Taylor expansion of $\log (1-w)$ in powers of $w$ we conclude that $-\log z \geq 1-z$ and

$$
\begin{aligned}
-\log y & =(1-y)+(1-y)^{2} / 2+\cdots \\
& <(1-y)\left[1+(3 / 4) / 2+(3 / 4)^{2} / 3+\cdots\right] \\
& =(8 / 3) \log 2(1-y) .
\end{aligned}
$$

Expanding $G(y, z)=-\log (z-y)+\log (1-z y)$ in powers of $(1-z)$ gives

$$
G(y, z)=(1+y)\left(\frac{1-z}{1-y}\right)+\sum_{k=2}^{\infty}\left(\frac{1-(-1)^{k} y^{k}}{k}\right)\left(\frac{1-z}{1-y}\right)^{k} .
$$

Thus, with $c=(16 / 3) \log 2$ we have

$$
\begin{aligned}
& (\log |z|)^{-1} G(y, z) \log y<c\left(\frac{1-y}{1-z}\right) \sum_{n=1}^{\infty} \frac{1}{n}\left(\frac{1-z}{1-y}\right)^{n} \\
& =c \sum_{n=0}^{\infty} \frac{1}{(n+1)}\left(\frac{1-z}{1-y}\right)^{n} \leq c \sum_{n=0}^{\infty} \frac{1}{(n+1)}\left(\frac{1-z}{1-z^{2}}\right)^{n} \\
& =c \sum_{n=0}^{\infty} \frac{1}{(n+1)}\left(\frac{1}{1+z}\right)^{n}<c \sum_{n=0}^{\infty}(n+1)^{-1}\left(\frac{2}{3}\right)^{n}=\frac{3}{2} \log 3 .
\end{aligned}
$$

Finally, turning to Case 4 we have $(-\log |z|)^{-1} G(y, z) \leq G(y,|z|) /(1-|z|)$ and we may assume $z=|z|$. By (2.4)

$$
G(y, z) \leq\left(\frac{5}{4}\right) \sum_{n=1}^{\infty} n^{-1}\left(\frac{1-z}{1-y}\right)^{n} .
$$

Thus

$$
G(y, z) /(1-z) \leq \frac{5}{3}+\left(\frac{5}{3}\right) \sum_{n=1}^{\infty}(n+1)^{-1}\left(\frac{2}{3}\right)^{n}=\frac{5}{2} \log 3 .
$$


Multiplying both sides by $-\log y$ completes the proof.

\section{Proof of Theorem 1.1 .}

We begin with the following key lemma which enables us to assume without loss of generality that both $G(x, y)$ and $G(y, z)$ are large.

Lemma 3.1. Let $\beta>0$ and suppose the inequality

$$
G(x, z)^{-1} G(x, y) G(y, z) \leq c[G(x, y)+G(y, z)]+c
$$

has been proved for a given domain $D$ with $c=c(D) \geq 1$ and all distinct points $x, y$, and $z$ in $D$ which also satisfy

$$
\min \{G(x, y), G(y, z)\} \geq \beta .
$$

Then (3.1) holds for all distinct $x, y$, and $z$ in $D$ with $c$ replaced by $c+2 \beta$. Proof. Fix $x, y$, and $z$ in $D$ and suppose that (3.2) is false.

Case 1. $\max \{G(x, y), G(y, z)\} \geq \beta$.

By symmetry we may assume without loss of generality that $G(x, y)<\beta$ and $G(y, z) \geq \beta$. We will then show that

$$
G(x, z)^{-1} G(x, y) G(y, z) \leq(c+\beta) G(y, z)+(c+\beta) .
$$

Let $A=\{v: G(v, y) \geq \beta\}$ and let $\omega$ denote the Brownian hitting distribution on $A$ under $P_{x}$, i.e.,

$$
\omega(d v)=P_{x}\left(B\left(\tau_{A} c\right) \in d v, \tau_{A} c<\tau\right) .
$$

Since $G(\cdot, y)$ is bounded and harmonic on $D \cap A^{c}$, we have

$$
G(x, y)=E_{x} G\left(B\left(\tau_{A} c \wedge \tau\right), y\right)=\int_{\partial A} G(v, y) \omega(d v)=\beta \omega(A) .
$$

Now for any $v \in \partial A$ we have by (3.1) (which holds, by assumption, for the triple $v, y, z)$,

$$
\beta G(y, z) \leq c G(y, z) G(v, z)+(\beta+c) G(v, z) .
$$

Integration with respect to $\omega(d v)$ over $\partial A$ gives

$$
\begin{aligned}
G(x, y) G(y, z) & =\beta \omega(A) G(y, z) \\
& \leq c G(z, y) \int_{\partial \cdot A} G(v, z) \omega(d v)+(\beta+c) \int_{\partial A} G(v, z) \omega(d v) .
\end{aligned}
$$

But

$$
\int_{\partial A} G(v, z) \omega(d v)=E_{x} G\left(B\left(\tau_{A} c \wedge \tau\right), z\right) \leq G(x, z) .
$$

Substituting this in the inequality above and then dividing through by $G(x, z)$, we obtain (3.3).

Case 2. $\max \{G(x, y), G(y, z)\}<\beta$.

We may repeat the argument just given, only using $c+\beta$ in place of $c$ in (3.4). The proof of Lemma 3.1 is complete. 
Next, we show that there exists a constant $\beta=\beta(D, \eta)$ such that

$$
|u-v|>\eta / 8 \Rightarrow G(u, v)<\beta .
$$

To see this, we may assume that $D^{c}$ contains an unbounded component, by performing a suitable inversion if necessary. Therefore the probabilistic formula $G(u, v)=E_{u} \log \left(|v-B(\tau)||u-v|^{-1}\right)$ is valid. We may assume without loss of generality that $u=0$. Define the Brownian maximal function by $B^{*}(\tau)=$ $\sup \{|B(t)|: 0 \leq t<\tau\}$. The results of [5] imply that $B^{*}(\tau)$ has a finite $p$ th moment for each $0<p<1 / 2$. To estimate this moment, note that the circular symmetrization, $D^{*}$, of $D$ with respect to the origin is contained in the plane minus a half-line on the negative $x$-axis of the form $\left(-\infty, x_{0}\right]$. Let $\sigma$ be the hitting time of Brownian motion to this half-line. Then using the results of [2] on the behavior of harmonic measure under symmetrization, as in [5, p. 199], we obtain

$$
E_{0}\left[B^{*}(\tau)\right]^{p} \leq E_{0}\left[B^{*}(\sigma)\right]^{p} \leq C,
$$

where $C=C\left(p, x_{0}\right)$, provided $0<p<1 / 2$. Thus, for any such $p$,

$$
\begin{aligned}
G(0, v) & <(1 / p)|v|^{-p} E_{0}|v-B(\tau)|^{p} \leq(1 / p)|v|^{-p}\left\{|v|^{p}+E_{0}\left[B^{*}(\tau)\right]^{p}\right\} \\
& \leq(1 / p)\left(C+|v|^{p}\right)|v|^{-p},
\end{aligned}
$$

and statement (3.5) follows.

Remark 3.2. If $|D|<\infty$, one may estimate more explicitly as follows:

$$
\begin{aligned}
G(0, v) & <(1 / 2)|v|^{-2} E_{v}|B(\tau)|^{2}=1 / 2+|v|^{-2} E_{v} \tau \\
& \leq 1 / 2+c|D||v|^{-2}
\end{aligned}
$$

where $c$ is an absolute constant. (See, e.g., [10, Lemma 2.1] for the last inequality.) Thus in this case we may choose $\beta$ depending only on $|D|$ and $\eta$. (We shall ultimately need to increase the value of $\beta$ further for other purposes below.)

To prove (1.3) we may assume $y=0$ and $|z| \geq|x|=x$. By Lemma 3.1 we may further assume $\min \{G(x, 0), G(0, z)\} \geq \beta$ and, therefore

$$
0<x<\eta / 8 \text {. }
$$

Let $B=B(0 ; 2 x)$ and $B^{\sim}=B(0,4 x)$. It follows from the maximum principle and the connectivity of $D$ that for any $\lambda>0$ the set $\{w: G(w, z)>\lambda\}$ is connected. Taking $\lambda=G(0, z) / 2$ shows that it is possible to find a curve $\Gamma$ connecting 0 and $z$ such that

$$
G(w, z) \geq G(0, z) / 2, \quad w \in \Gamma .
$$

(Refer to Figure 1.)

It is now time to specify the final value of $\beta$. Take $\beta$ to satisfy $\beta>\beta_{0}$, where $\beta_{0}$ is the value at the point $(1,0)$ of the Green function of the slit plane $\mathbf{C} \backslash[-4,-2]$, in addition to the earlier constraints placed upon $\beta$. 


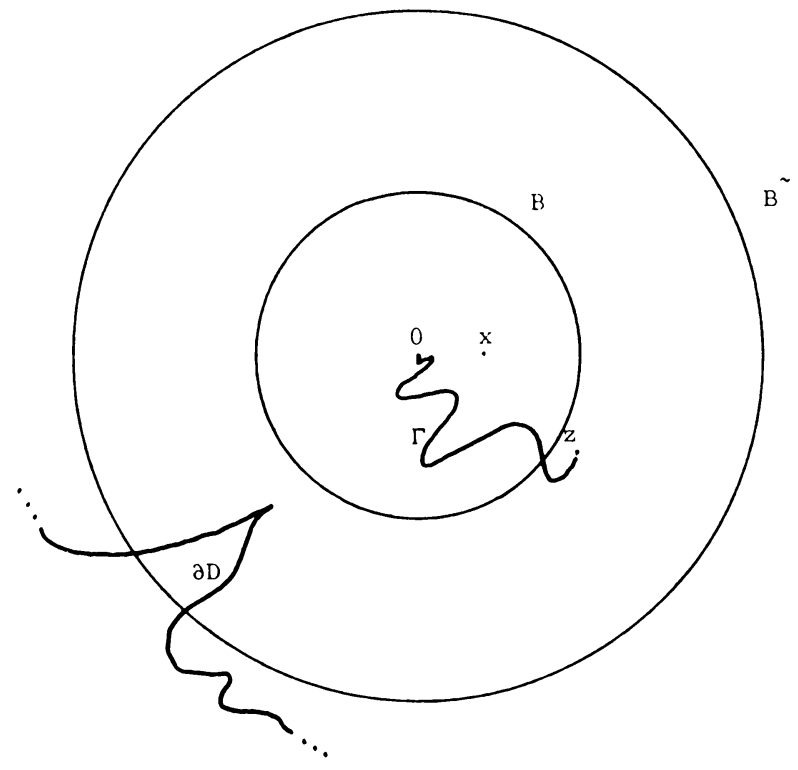

FIGURE 1

Claim. If $K$ is a connected component of $D^{c}$ then $G(0, x)>\beta$ implies that $K$ does not meet $B$, i.e., $B \subset D$.

Leaving aside, for a moment, the verification of the claim, let us show how (1.3) follows. Indeed, we will prove $G(x, z)^{-1} G(x, 0) G(0, z) \leq c G(x, 0)$, a stronger inequality. Let $\mu$ denote the hitting time to $\Gamma$, and $\nu$ the exit time from $B$, of Brownian motion. Then $P_{x}(\mu<\tau) \geq P_{x}(\mu<\nu)$. We may use the theory of symmetrization to estimate the latter probability from below. Let $\mu^{*}$ denote the hitting time of Brownian motion to the segment $[-x, 0]$. Then by [2, Theorem 7] and the fact that $|z| \geq x$, we have

$$
P_{x}(\mu<\nu) \geq \inf _{|\xi|=x} P_{\xi}\left(\mu^{*}<\nu\right) \stackrel{d}{=} p_{0}>0,
$$

where $p_{0}$ is an absolute constant. By superharmonicity of $G$,

$$
G(x, z) \geq E_{x} G(B(\mu \wedge \tau), z)=E_{x}[G(B(\mu), z) ; \mu<\tau] \geq p_{0} G(0, z) / 2 .
$$

Thus we conclude that $G(x, z)^{-1} G(x, 0) G(0, z) \leq\left(2 / p_{0}\right) G(x, 0)$, which is the desired result.

There remains only to prove the claim, which we do by contradiction. Suppose $K \cap B$ were not empty, say $w_{1} \in K \cap B$. By (3.6) and the definition of $\eta$ we have that $K$ cannot be contained in $B^{\sim}$ (else its diameter would be too small) so we can find a point $w_{2}$ in $K$ which lies outside the disc $B^{\sim}$. Since $K$ is connected, there is some curve $\gamma$ contained in $K$ which connects $w_{1}$ to $w_{2}$. It follows that the circular symmetrization $D^{*}$ is contained in the domain $R$ formed by removing the segment $[-4 x,-2 x]$ from the plane. By dilation invariance of $G_{R}$ we then have $G_{R}(x, 0)=\beta_{0}$. Then, combining Theorem 5 


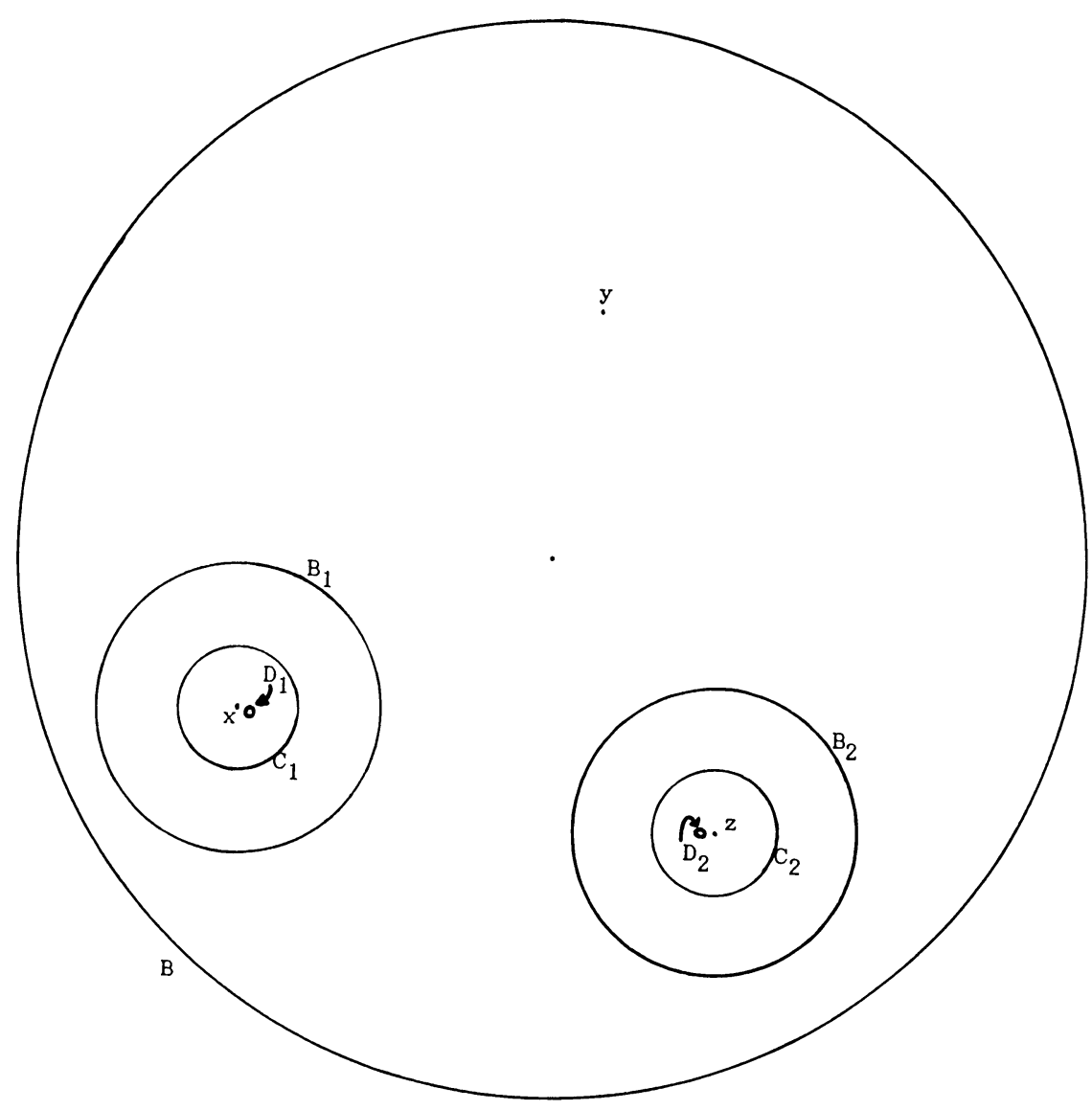

FIGURE 2

and the Corollary of Proposition 5 in [2], we have

$$
G(x, 0) \leq G_{D^{*}}(x, 0) \leq G_{R}(x, 0)=\beta_{0}<\beta,
$$

a contradiction which completes the proof of Theorem 1.1.

\section{Proof of Theorem 1.4}

Let $h$ be a positive harmonic function on $D$. In [7] Chung shows how to reduce the CGT to the "gauge theorem" of [19] whenever the following two conditions hold true:

(A) $P_{x}^{h}(\tau<\infty)=1$, and, for each $\varepsilon>0$ there exists $\delta=\delta(\varepsilon)>0$ such that

(B) $\int_{E} h(x)^{-1} G(x, y) h(y)|V(y)| d y<\varepsilon$ for each open subset $E$ of $D$ satisfying $|E|<\delta$.

Since the gauge theorem of [19] is true in our setting, we need only verify (A) and (B) to prove Theorem 1.4. (Alternatively, one may follow the proof of [15, Theorem 2].) However, Chung's argument that (A) and (B) imply CGT is given 
under the (inessential) assumption that $D$ be bounded Lipschitz. Therefore, before proving (A) and (B), we will indicate the necessary changes in Chung's argument.

The crux of the matter is the following: For a positive number $s$ let $D_{0}=$ $\left\{x \in D: \operatorname{dist}\left(x, D^{c}\right)>s / 2\right\}$. Note that $D_{0}$ has compact closure since we are assuming that $D$ has finite area. The open set $\left\{x \in D: \operatorname{dist}\left(x, D^{c}\right)<s\right\}$ has finitely many connected components since each must contain some disc of radius $s / 2$. By joining these components with very thin tubes contained in $D$, it is easy to construct a set $E$ with the following properties:

$E$ is open and connected,

$|E|$ may be made small by taking $s$ to be small,

$E$ contains $\partial D_{0}$, and

$D_{0}$ contains $(\partial E) \cap D$.

Our proof of the next result essentially follows Chung, except for the use of Theorem 1.1.

Lemma 4.1. There exists $a$ constant $b$ depending only on $s$ and $D$ such that for any $h>0$ harmonic on $D$ we have

$$
P_{x}^{h}\left(\tau_{E}=\tau\right) \geq b>0, \quad x \in \partial D_{0} .
$$

Proof. Fix a reference point $z_{0}$ in $D_{0}$. Let $\partial_{m} D$ denote the minimal Martin boundary of $D$ and $K(x, y)$ the Martin kernel normalized by $K\left(z_{0}, \cdot\right) \equiv 1$ on $\partial_{m} D$. Recall that $\partial_{m} D$ is compact in the Martin topology and $K$ is continuous on $\partial D_{0} \times \partial_{m} D$ in the (euclidean) $\times($ Martin) topology. (See, e.g., [16, Lemma 12.3].)

It is sufficient to prove (4.5) for $h(\cdot)=K(\cdot, z), z \in \partial_{m} D$. Now

$$
P_{x}^{z}\left(\tau_{E}=\tau\right)=K(x, z)^{-1}[K(x, z)-f(x, z)]
$$

where

$$
f(x, z)=E_{x}\left[K\left(B\left(\tau_{E}\right), z\right) ; \tau_{E}<\tau\right] .
$$

For any point $y$ in the closure of $D_{0}$ we have for some sequence $z_{n}$

$$
\begin{aligned}
K(y, z) & =\lim _{z_{n} \rightarrow z} G\left(y, z_{n}\right) / G\left(z_{0}, z_{n}\right) \\
& \leq \limsup _{n \rightarrow \infty} c\left[G\left(y, z_{n}\right) / G\left(z_{0}, y\right)+1\right]+c G\left(z_{0}, y\right)^{-1} \\
& =c+c G\left(z_{0}, y\right)^{-1}
\end{aligned}
$$

where the inequality follows from Theorem 1.1. The latter quantity is bounded as $y$ ranges over the closure of $D_{0}$. Since $B\left(\tau_{E}\right) \in(\partial E) \cap D$ on $\left\{\tau_{E}<\tau\right\}$, it follows from (4.4) and the dominated convergence theorem that $f(x, \cdot)$ is continuous on $\partial_{m} D$ for each $x$ in $D$; moreover, $f(\cdot, z)$ is harmonic, hence continuous on $E$, for each $z$ in $\partial_{m} D$. Since $\partial_{m} D$ and the closure of $D_{0}$ are disjoint compact sets, it follows that $f$ is continuous on $\partial D_{0} \times \partial_{m} D$. 
Finally, $K(x, z)-f(x, z)$ is strictly positive on $E$ for each $z$ in $\partial_{m} D$. (This follows from its harmonicity on $E$, since $f$ is bounded on $E$ while $K$ is unbounded there-see [10, Lemma 2.2].) The desired result now follows by continuity.

The remainder of Chung's argument, involving a sequence of hitting times, goes through without any essential change. (His notation is somewhat different from ours.)

There remains to verify (A) and (B). Condition (A) is immediate from the main result of [10] for any arbitrary domain of finite area. As for (B), first choose $r>0$ so small that

$$
\int_{B(x ; r)} G(x, y)|V(y)| d y<\frac{\varepsilon}{2 c}, \quad x \in D,
$$

where $c$ is as in Theorem 1.1, and we assume $G$ is extended to be equal to zero off $D$. For $x, y$ in $D,|x-y|>r$, there is a constant $M$ such that $G(x, y) \leq M$. (See (3.5) above.) By (1.7) it is possible to write $V=V_{1}+V_{2}$ where $V_{2}$ is bounded and $\left\|V_{1}\right\|_{1}$ is as small as desired. Then, by Corollary 1.2,

$$
\begin{array}{rl}
\int_{E} h(x)^{-1} & G(x, y) h(y)|V(y)| d y \\
& \leq c \int_{E} G(x, y)|V(y)| d y+c \int_{E}|V(y)| d y \\
& \leq \varepsilon / 2+(M+1) c \int_{E}|V(y)| d y \\
& \leq \varepsilon / 2+c(M+1)|E|\left\|V_{2}\right\|_{\infty}+c(M+1)\left\|V_{1}\right\|_{1} .
\end{array}
$$

The latter expression may be made smaller than $\varepsilon$ by first taking $\left\|V_{1}\right\|_{1}$, and then $|E|$, to be sufficiently small.

\section{AN EXAMPLe}

Let $x, y$, and $z$ be the vertices of an equilateral triangle centered at 0 and $B=B(0 ; R)$ a disc centered at 0 and large enough to contain $x, y$ and $z$; say, $R>2|x|$ for definiteness. We will construct a domain $D$ of the form $D=B \backslash\left(D_{1} \cup D_{2}\right)$, where $D_{1}$ and $D_{2}$ are small discs such that the constants in (1.3) can be made as large as desired by taking $R$ large and the $D_{i}$ very small and very close to $x$ and $z$. The discs $B_{i}$ and $C_{i}$ shown in Figure 2 are not part of the description of $D$, but will be used in the argument. The intuition behind the example is that the value of each of $G(x, y)$ and $G(y, z)$ is significantly affected (lowered) by only one of the $D_{i}$, whereas $G(x, z)$ is affected by both.

Let $B_{1}=B(x ;|x| / 2)$ and $B_{2}=B(z ;|z| / 2)$ and choose discs $C_{i}$ concentric with the $B_{i}$ such that

$$
\operatorname{diam}\left(C_{i}\right)<\operatorname{diam}\left(B_{i}\right) / 2, \quad i=1,2,
$$

and so small that

$$
G_{B}(u, v) \leq 2 G_{B \backslash\left(C_{1} \cup C_{2}\right)}(u, v), \quad u, v \in B \backslash\left(B_{1} \cup B_{2}\right) .
$$


The following inequalities, in which $c$ stands for a constant depending on $R$ and the choice of the $C_{i}$, are all immediate consequences of Harnack's inequality:

$$
\begin{aligned}
& G_{B \backslash\left(C_{1} \cup C_{2}\right)}\left(u_{1}, v\right) \leq c G_{B \backslash\left(C_{1} \cup C_{2}\right)}\left(u_{2}, v\right), \\
& v \in \operatorname{cl}\left(B_{2}\right)\left(\text { resp. } B_{1}\right), u_{1}, u_{2} \in \partial B_{1}\left(\text { resp. } \partial B_{2}\right), \\
& \left.G_{B \backslash\left(C_{1} \cup C_{2}\right)}\left(u_{1}, y\right) \leq c G_{B \backslash\left(C_{1} \cup C_{2}\right)}\left(u_{2}, y\right), \quad u_{i} \in \partial B_{1} \text { (resp. } \partial B_{2}\right) \text {, } \\
& \frac{1}{c} G_{B}(x, y) \leq G_{B}(u, y) \leq c G_{B}(x, y), \quad u \in \partial B_{1}, \quad \text { and } \\
& \frac{1}{c} G_{B}(z, y) \leq G_{B}(v, y) \leq c G_{B}(z, y), \quad v \in \partial B_{2} .
\end{aligned}
$$

Notice that $C$ need not increase as $R$ increases or as the diameters of the $C_{i}$ decrease.

Let $D_{i}(i=1,2)$ be small discs contained in $C_{i}$ with $x \notin D_{1}$ and $z \notin D_{2}$. Let $p_{1}=P_{x}\left(B(t)\right.$ does not hit $D_{1}$ before leaving $\left.B_{1}\right)$, and $p_{2}=P_{z}(B(t)$ does not hit $D_{2}$ before leaving $B_{2}$ ). Then the $p_{i}$ can be made as small as desired by taking $D_{1}$ and $D_{2}$ sufficiently close to $x$ and $z$, respectively.

Let $D=B \backslash\left(D_{1} \cup D_{2}\right)$ and $G=G_{D}$.

By (5.2) and monotonicity of the Green function in its domain,

$$
G_{B}(u, v) \leq 2 G(u, v), \quad u, v \in B \backslash\left(B_{1} \cup B_{2}\right) .
$$

Next, we prove the following inequalities, in which $c$ is the constant of $(5.3)$ :

$$
\begin{aligned}
& \left(p_{1} / 2 c^{2}\right) G_{B}(x, y) \leq G(x, y) \leq c^{2} p_{1} G_{B}(x, y), \\
& \left(p_{2} / 2 c^{2}\right) G_{B}(y, z) \leq G(y, z) \leq c^{2} p_{2} G_{B}(y, z), \quad \text { and } \\
& \left(p_{1} p_{2} / 2 c^{4}\right) G_{B}(x, z) \leq G(x, z) \leq p_{1} p_{2} c^{4} G_{B}(x, z) .
\end{aligned}
$$

Let $\mu$ and $\nu$ be the Brownian exit times from $B_{1}$ and $B_{2}$, respectively. Also choose and fix arbitrary points $x_{i} \in \partial B_{i} \quad(i=1,2)$. Then

$$
\begin{aligned}
G(x, y) & =E_{x} G(B(\mu \wedge \tau), y) \geq\left(p_{1} / c\right) G\left(x_{1}, y\right) \\
& \geq\left(p_{1} / 2 c\right) G_{B}\left(x_{1}, y\right) \geq\left(p_{1} / 2 c^{2}\right) G_{B}(x, y),
\end{aligned}
$$

which is the left-hand side of (5.5). For the other side we have

$$
\begin{aligned}
G(x, y) & =E_{x} G(B(\mu \wedge \tau), y) \leq c p_{1} G\left(x_{1}, y\right) \\
& \leq c p_{1} G_{B}\left(x_{1}, y\right) \leq c^{2} p_{1} G_{B}(x, y) .
\end{aligned}
$$

The proof of $(5.6)$ is the same.

To obtain the right-hand side of (5.7) we employ the same reasoning twice in succession:

$$
\begin{aligned}
G(x, z) & =E_{x} G(B(\mu \wedge \tau), z) \leq c p_{1} G\left(x_{1}, z\right) \leq c p_{1} E_{z} G\left(x_{1}, B(\nu \wedge \tau)\right) \\
& \leq c^{2} p_{1} p_{2} G\left(x_{1}, x_{2}\right) \leq c^{2} p_{1} p_{2} G_{B}\left(x_{1}, x_{2}\right) \\
& \leq c^{4} p_{1} p_{2} G_{B}(x, z) .
\end{aligned}
$$

The proof of the other side of (5.7) is similar. 
Now, combining (5.5)-(5.7), we have

$$
\begin{aligned}
G(x, z)^{-1} G(x, y) G(y, z) & \geq\left(1 / 4 c^{8}\right) G_{B}(x, z)^{-1} G_{B}(x, y) G_{B}(y, z) \\
& =\left(1 / 4 c^{8}\right) G_{B}(x, y),
\end{aligned}
$$

since $G_{B}(x, y)=G_{B}(y, z)=G_{B}(x, z)$ by symmetry.

The desired conclusion follows since $G_{B}(x, y)$ can be made arbitrarily large by taking $R$ large, and the ratio $G_{B}(x, y) / G(x, y)$ can be made arbitrarily large by ensuring that $p_{1}$ is small.

\section{REFERENCES}

1. M. Aizenmann and B. Simon, Brownian motion and Harnack inequality for Schrödinger operators, Comm. Pure Appl. Math. 35 (1982), 209-273.

2. A. Baernstein, Integral means, univalent functions and circular symmetrization, Acta Math. 133 (1974), 139-169.

3. R. Bañuelos, On an estimate of Cranston and McConnell for elliptic diffusions in uniform domains, Probab. Theor. Related Fields 76 (1987), 311-323.

4. R. Bañuelos and B. Davis, Heat kernel, eigenfunctions, and conditioned Brownian motion in planar domains, J. Funct. Anal. 84 (1989), 188-200.

5. D. L. Burkholder, Exit times of Brownian motion, harmonic majorization, and Hardy spaces, Adv. in Math. 26 (1977), 182-205.

6. K. L. Chung, The lifetime of conditional Brownian motion in the plane, Ann. Inst. H. Poincare Sect. A (N.S.) 20 (1984), 349-351.

7. K. L. Chung, The gauge and conditional gauge theorem, Séminaire de Probabilités XIX, Lecture Notes in Math., vol. 1123, Springer-Verlag, Berlin, 1983/84, pp. 496-503.

8. K. L. Chung and M. Rao, Feynmann-Kac functional and Schrödinger equation, Sem. Stochastic Processes, Birkhäuser, Boston, Mass., 1981.

9. M. Cranston, Lifetime of conditioned Brownian motion in Lipschitz domains, Z. Wahrsch. Verw. Gebiete 70 (1985), 335-340.

10. M. Cranston and T. R. McConnell, The lifetime of conditioned Brownian motion, Z. Wahrsch. Verw. Gebiete 65 (1983), 1-11.

11. M. Cranston, E. Fabes, and Z. Zhao, Conditional gauge and potential theory for the Schrödinger operator, Trans. Amer. Math. Soc. 307 (1988), 171-194.

12. B. Davis, Conditioned Brownian motion in planar domains, Duke Math. J. 57 (1988), 397421.

13. R. D. DeBlassie, The lifetime of conditioned Brownian motion in certain Lipschitz domains, Probab. Theor. Related Fields 75 (1987), 55-65.

14. N. Falkner, Feynmann-Kac functionals and positive solutions of $\frac{1}{2} \Delta u+q u=0, \mathrm{Z}$. Wahrsch. Verw. Gebiete 65 (1983), 19-33.

15. __ Conditional Brownian motion in rapidly exhaustible domains, Ann. Probab. 15 (1987), 1501-1514.

16. L. L. Helms, Introduction to potential theory, Wiley, New York, 1969.

17. P. J. Myrberg, Über die existenz der Greenschen funcktionen auf einer Riemannschen Fläche, Acta Math. 61 (1933), 39-79.

18. T. Salisbury, A Martin boundary in the plane, Trans. Amer. Math. Soc. 293 (1986), 623-642.

19. Z. Zhao, Conditional gauge with unbounded potential, Z. Wahrsch. Verw. Gebiete 65 (1983), 13-18. 